\section{Das Lignin des mit Alkalicarbonat aufgeschlossenen Strohes.}

\author{
Von F. PASChKE-Danzig.
}

(Eingeg. 12.j8. 1921.)

In Nr. 50 (S. 285) dieser Zeitschrift (Jahrg. 34) haben Ernst Beckmann und seine Mitarbeiter eine sehr interessante Arbeit über "Lignin aus Winterroggenstroh" veröffentlicht. In dieser Abhandlung beschreiben sie auch eine Bestimmung des Äquivalentgewichtes des Lignins. Diese Bestimmung führten sie derart aus, daß ein ÜberschuB von Lignin in 0,1-n Natronlauge gebracht wurde, und daß in der vom ungelösten Lignin getrennten Flüssigkeit der Natriumgehalt bestimmt wurde. Daraus fanden sie das Äquivalentgewicht 462 .

Auch ich beschäftigte mich seit einiger Zeit mit dem Studium des Lignins und habe bereits über Stroblignin veröffentlicht im "Wochenblatt für Papierfabrikation" und zwar in Nr. 16, Jahrg. 1920, Nr. 33, Jahrg. 1920 und Nr. 23, Jahrg. 1921. In Nr. 33 habe ich auch eine Bestimmung des Äquivalentgewichts beschrieben, die auch auf der Bildung von Natriumlignat beruht und die im Gegensatz zu der obenerwähnten Methode mit einem Überschuß von Natronlauge ausgeführt wurde.

Es erscheint interessant, beide Bestimmungen $\mathrm{zu}$ vergleichen, und es sei daher gestattet, die bereits veröffentlichte Beschreibung der letztgenannten Bestimmung hier $\mathrm{zu}$ wiederholen:

Es war Stroh mit Soda aufgeschlossen worden und das Lignin der Ablauge durch Salzsäure ausgefällt worden. Dieses Lignin wurde durch wiederholtes Auflösen in Sodalösung und Ausfällen mit Salzsäure gereinigt und gab alsdann das Ausgangsmaterial für die nachfolgend beschriebene Untersuchung. Die Äquivalentgewichtsbestimmung bestand darin, daß Lignin in überschüssiger Natronlauge aufgelöst wurde und daß alsdann die so entstandene Lösung und die angewandte Lauge titrimetrisch verglichen wurden.

Die Messungen wurden derart ausgeführt, daß bei jeder Titration die Indikatoren Phenolphthalein und Methylorange nacheinander benutzt wurden.

\begin{tabular}{cccc}
\hline Indikator & $10 \mathrm{ccm}$ Lauge & $\begin{array}{c}10 \mathrm{ccm} \text { Lignin- } \\
\text { lösung } \\
(1,0 \mathrm{~g} \text { in } 100 \mathrm{ccm} \\
\text { Lauge })\end{array}$ \\
\hline Phenolphthalein & $9,7 \mathrm{ccm}{ }_{10}^{\mathrm{n}}$ Säure & $6,9 \mathrm{ccm}{ }_{10}^{\mathrm{n}}$ Säure \\
Methylorange & $0,9 \mathrm{~cm}{ }_{10}^{\mathrm{n}}$ Säure & $3,7 \mathrm{ccm}{ }_{10}^{\mathrm{n}}$ Säure
\end{tabular}

Es zeigte sich also, daß das Lignin in der Lauge sich so verhält wie aufgelöste Kohlensäure, so daß man daher das Lignin mit der Kohlensäure vergleichen kann. Der Methylorange-Titer war durch das Auflösen des Lignins von $0,9 \mathrm{ccm}$ auf $3,7 \mathrm{ccm}$ gestiegen, also um eine Differenz von $2,8 \mathrm{~cm}$

Diese $2,8 \mathrm{~cm}{ }_{10}^{n}$. :äure entsprechen einer scheinbaren Menge Kohlensäure von $123,2 \mathrm{mg}$ in $100 \mathrm{~cm}$ Lauge. Die beobachtete Änderung des Titers ist aber nicht durch 12:3, ing Kohlensäure, sondern durch $1000 \mathrm{mg}$ Lignin hervorgerufen worden. Aus diesem Verhältnis errechnet sich das Äquivalentgewicht des Lignins zu 357. Beckmann und seine Mitarbeiter haben ein höheres Äquivalentgewicht gefunden, nämlich 462. Sie vermuten, dal3 dieses hohe Gewicht dadurch hervorgerufen ist, da $B$ kolloidal gelöstes Lignin durchs Filter gegangen ist. Diese Vermutung ist gerechtfertigt.

Tatsächlich konnte ich feststellen, daß3 Jignin in erheblichem Maße in destilliertem Wasser kolloidal löslich ist und aus dieser Lösung durch Metallsalzlösungen sowje durch Lisungen von Chlorbarium oder (hlorealeium gefällt wird ${ }^{1}$ )

Das Beckmann-Lignin verlangt nach der Theorie das Aquivalentgewicht 38\%,3, so daß3 also der theoretische Wert von Beckman nur um 6, $6 \%$ grölier ist als der von mir gefundene (357).

Das Sodaaufschluk-Lignin, welches das Versuchsmaterial geliefert hatte, war von folgender Zusammensetzung: $\mathrm{C} \cdots 64,8^{\circ} \% \mathrm{H}=6,16^{\circ}$ $\mathrm{O}=29,0^{\prime \prime}{ }_{11}$; daraus errechnete $\operatorname{sich}\left(\mathrm{C}_{2} \mathrm{H}_{: 31} \mathrm{O}_{49} \quad 499,4\right.$ als Bruttoformel $\left.{ }^{2}\right)$ Nimmt man an, da 3 im Natriumlignat auf 20 C-Atome 1 Na-Atom kommt, so berechnet sich für das ligniı des Verfassers das Äquivalentgewirht zu $\begin{gathered}499,4 ; 20 \\ 27\end{gathered}$

Hier ist die Uthereinstimmung von Theorie und Beobachtung noch besser, Differenz $3.5^{\circ}$

1) Pasc ske, Wochenblatt für Papierfabrikation, Jahrg. 1920, S. 2322. Angew. Chemie 1921. Nr. 73.
Ein Vergleich der Analysen und Bruttoformeln beider in Rede stehenden Lignine zeigt, daß beide sich erheblich voneinander unterscheiden und zwar besonders im Sauerstoffgehalt. Das Beckmann Lignin hat mehr Sauerstoff. Es scheint jedoch dieser Unterschied auf einer gewissen Gesetzmäßigkeit zu beruhen; denn durch Hinzufügung eines Sauerstoffatomes zur Bruttoformel des Sodaaufschluß lignins entsteht eine hypothetische Bruttoformel, die in ihrer prozentuellen Zusammensetzung dem Beckmann-Lignin sehr nahe kommt.

\begin{tabular}{|c|c|c|c|}
\hline Bruttoformel & $\% \mathrm{C}$ & $\% / 0 \mathrm{H}$ & $\%$ \\
\hline 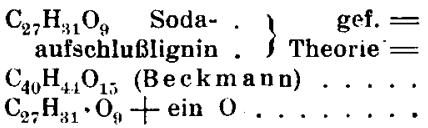 & $\begin{array}{l}64,8 \\
64,9 \\
62.8 \\
62,9\end{array}$ & $\begin{array}{l}6,2 \\
6,3 \\
5,8 \\
6,1\end{array}$ & $\begin{array}{l}29,0 \\
28,8 \\
\mathbf{3 1}, \mathbf{4} \\
\mathbf{3 1 , 0}\end{array}$ \\
\hline
\end{tabular}

Das Resultat dieser Betrachtungen kann wie folgend zusammengefaßt werden

Die beiden Strohlignine des Beckmann-Aufschlusses und des Sodaaufschlusses unterscheiden sich wesentlich in ihrem Sauerstoffgehalt. Dieser Unterschied zeigt jedoch eine gewisse Gesetzmäßigkeit.

Im Natriumlignat kommt auf 20 C-Atome 1 Natriumatom, unabhängig vom Sauerstoffgehalt des , betreffenden Lignins. Unmittelbar vor der Veröffentlichung dieser Arbeit habe ich nochmals einen Strohaufschluß mit Soda ausgeführt und das dabei gewonnene Lignin analysiert:

$0,2254 \mathrm{~g}$ gaben $0,5399 \mathrm{~g} \mathrm{CO}, 2$ und $0,1248 \mathrm{~g} \mathrm{H}_{2} \mathrm{O}$. Dies entspricht $\mathrm{C}=65,4^{\%} ; \mathrm{H}-6,2 \% ; \mathrm{O}=28,4^{\circ} ;$.

\begin{tabular}{l|c|c|c}
\hline & $0_{0} \mathrm{C}$ & $0 /_{0} \mathrm{H}$ & $0 / 0$ \\
\hline frühere Analyse $\ldots \ldots \ldots \ldots \ldots$ & 64,8 & 6,2 & 29,0 \\
neue Analyse $\ldots \ldots \ldots \ldots$ & 65,4 & 6,2 & 28,4
\end{tabular}

Nach der neuen Analyse ist der Sauerstoffgehalt etwas kleiner und der Kohlenstoffgehalt etwas größer, so daß die Analyse besser auf die Bruttoformel $\mathrm{C}_{40} \mathrm{H}_{45} \mathrm{O}_{18}$ paßt. Wie folgende Zusammenstellung zeigt:

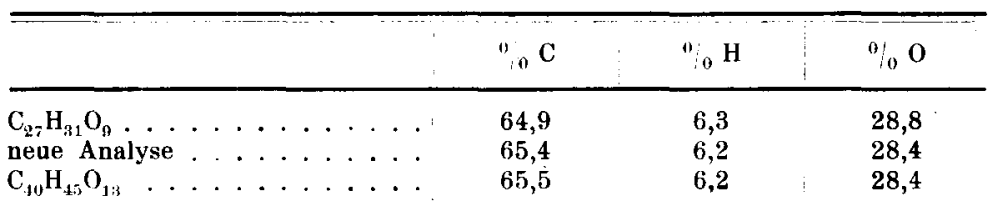

Auch durch die zweite Analyse wird das vorher Gesagte bestätigt Ein Vergleich der neuen Formel $\mathrm{C}_{40} \mathrm{H}_{45} \mathrm{O}_{13}$ mit der Formel von Beck mann und seinen Mitarbeitern $\mathrm{C}_{1} \mathrm{H}_{1} \mathrm{O}_{15}$ zeigt deutlich, daß die beiden Lignine sich hauptsächlich durch ihren Sauerstoffgehalt unterscheiden.

[A. 192.]

\section{Die Erhaltung der Elastizität im Kautschuk durch künstliche Anregung kolloider Tätigkeit} mittels Alkalien und anderer quellender Mittel.

$$
\text { Von Dr. Rudohe DITMAR-Graz. }
$$

(Eingeg. 16.' 8.1921.$)$

Verschiedene Frscheinungen am „alternden“ Kautschuk und die Wirkung einiger kautschukkonservierender Mittel führten mich au den Gedanken, daß die Elastizität eines organischen Kolloids (Kautschuk, Leder, Cellulose usw.) auf der Fähigkeit beruht, zu quellen und zu entquellen, zu dispergieren und zu aggregieren. Solange einem organischen Kolloid diese Potenz innewohnt, ist es elastisch Durch fortwährende Übung, indem man dem Kolloid Gelegenheit gibt zu quellen und wieder $\mathrm{zu}$ entquellen, erhält man diese Potenz. Läßt man hingegen das Kolloid ruhen, nimmt man ihm mit anderen Worten die Gelegenheit, sich nach beiden Richtungen hin zu betätigen, dann schwindet die Elastizität und das Kolloid wird brächig.

Es ist eine bekannte 'Tatsache, daß Kautschuk im Gebrauche elastisch bleibt (im Pneu z. B., wenn man ihn fährt und bald dem Regen, bald der Trockenheit aussetzt), beim Liegen dagegen hart wird; Wäsche im Gebrauche bleibt weich und schmiegsam (in der Wäsche quillt sie, beim liegen entquillt sie wieder), Seide wird durch Lieoen brüchig, Schuhe werden durch langes Iagern hart, Celluloid spröde usw. Das tägliche Einschmieren der Schuhe mit Schuhcreme bewirkt nichts weiter als eine Fettquellung, welche beim Tragen wieder entquillt - durch diese Übung bleibt das Leder elastisch.

Was man beim Leder mit Creme erreicht, das suchen zahlreiche Patente auf mehr oder weniger umständliche Weise beim Kautschuk zu bewirken, so: 